\title{
Predicción de la fiabilidad de elementos no reparables dirigido a lámparas de alumbrado público
}

\author{
Prediction of the reliability of non-repairable elements directed to street \\ lighting lamps of street lighting
}

1 César Marcelo Gallegos Londoño https://orcid.org/0000-0002-8685-7501 Escuela Superior Politécnica de Chimborazo (ESPOCH), Facultad de Mecánica. Riobamba, Ecuador. cesar.gallegos@espoch.edu.ec

2 Félix Antonio García Mora https://orcid.org/0000-0001-5814-3694 Escuela Superior Politécnica de Chimborazo (ESPOCH), Facultad de Mecánica. Riobamba, Ecuador. felix.garcia@espoch.edu.ec

3 Alex Giovanny Tenicota García https://orcid.org/0000-0002-0707-7698 Escuela Superior Politécnica de Chimborazo (ESPOCH), Facultad de Mecánica.

Riobamba, Ecuador.

alex.tenicota@espoch.edu.ec

Artículo de Investigación Científica y Tecnológica
Enviado: $24 / 12 / 2021$
Revisado: $29 / 12 / 2021$
Aceptado: $18 / 01 / 2022$
Publicado:14/02/2022
DOI: https://doi.org/10.33262/ap.v4i1.179

Cítese: $\quad$ Gallegos Londoño, C. M., García Mora, F. A., \& Tenicota García, A. G. (2022). Predicción de la fiabilidad de elementos no reparables dirigido a lámparas de alumbrado público. AlfaPublicaciones, 4(1), 144-162. https://doi.org/10.33262/ap.v4i1.179

CONCIENCIA DIGITAL, es una Revista Multidisciplinar, Trimestral, que se publicará en soporte electrónico tiene como misión contribuir a la formación de profesionales competentes con visión humanística y crítica que sean capaces de exponer sus resultados investigativos y científicos en la misma medida que se promueva mediante su intervención cambios positivos en la sociedad. https://concienciadigital.org

La revista es editada por la Editorial Ciencia Digital (Editorial de prestigio registrada en la Cámara Ecuatoriana de Libro con No de Afiliación 663) www.celibro.org.ec 
Palabras claves: fiabilidad, probabilidades, equipos no reparables, distribuciones paramétricas
Keywords: reliability, probabilities, unrepairable equipment, parametric
Resumen

El presente estudio tiene como propósito determinar los pasos necesarios para el cálculo de los indicadores de fiabilidad en equipos no reparables, el estudio se lo realizó en una muestra de 250 lámparas de sodio de 100 watios destinadas al alumbrado público. Siendo el tiempo para la falla TTF el parámetro más importante para realizar un análisis de fiabilidad en equipos no reparables, (tiempos desde la instalación del equipo hasta la falla), en este caso los equipos no se pueden reparar, por consiguiente, deben ser reemplazados después del fallo, la recolección óptima de estos datos es fundamental para realizar un análisis correcto de los indicadores de fiabilidad. Se debe buscar cuál de las funciones paramétricas es la que mejor se ajusta a los datos recolectados, para esto es necesario hallar la Función $\mathrm{F}(\mathrm{t})$ que representa a la distribución paramétrica tiempo acumulado hasta la falla y compararla con la función no paramétrica o función empírica. Se deben calcular los parámetros de todas las distribuciones hipotéticas seleccionadas con los datos recolectados en este estudio. Para cada distribución se calcula el valor de prueba el que es comparado con el valor crítico de la prueba de ajuste de bondad de Kolmogorov-Smirnov, definiendo de esta manera si las hipótesis son rechazadas o no, el rechazo significa que el valor de la prueba es mayor que el valor crítico de la prueba, caso contrario las hipótesis son aceptadas y el valor más bajo decide la distribución paramétrica que mejor se ajusta a los datos de la muestra. Una vez seleccionada la distribución se puede estimar los indicadores de la fiabilidad como la función de probabilidad de fallas acumulada $\mathrm{F}(\mathrm{t})$, la Función de Supervivencia $\mathrm{R}(\mathrm{t})$ y la función de la tasa de fallos conocida también como función de riesgo $\lambda(\mathrm{t})$. El método proporcionará una herramienta muy útil para el cálculo de la fiabilidad en equipos reparables y su futura evaluación que proporcionará ideas para la toma de decisiones en función de la una mejora continua.

\section{Abstract}

The purpose of this study is to determine the necessary steps for the calculation of reliability indicators in non-repairable equipment, the study was carried out on a sample of 250 100-watt sodium lamps for public lighting. Being the time to failure TTF the most important parameter to perform a reliability analysis on non-repairable 
equipment, (time since the equipment installation until its failure), in this case the equipment cannot be repaired, therefore, it must be replaced. After the failure, the optimal data collection is essential to carry out a correct analysis of the reliability indicators. It is necessary to find which of the parametric functions is the one that fits the best the collected data, for this it is imperative to find the Function $\mathrm{F}(\mathrm{t})$ that represents the parametric distribution accumulated time to failure and compare it with the non-parametric function or empirical function. Parameters of all selected hypothetical distributions must be calculated with the data collected in this study. For each distribution, the test value is calculated, which is compared with the critical value of the KolmogorovSmirnov kindness fit test, therefor defining whether the hypotheses are rejected or not. If rejected, it means that the value of the test is greater than the critical value, otherwise the hypotheses are accepted, and the lowest value decides the parametric distribution that fits the best with the sample data. Once the distribution has been selected, reliability indicators can be estimated, such as the cumulative failure probability function $\mathrm{F}(\mathrm{t})$, the Survival Function $\mathrm{R}(\mathrm{t})$, and the failure rate function, also known as the risk function $\lambda(t)$. The method will provide a very useful tool for calculating the reliability of repairable equipment and its future evaluation, which will provide ideas for decision-making based on continuous improvement.

\section{Introducción}

El estudio de la fiabilidad proporciona información esencial en el desarrollo de las técnicas o modelos productivos, su enfoque se cimenta en la predicción de procesos basado en conceptos estadísticos. El análisis de la Fiabilidad y sus indicadores proporcionan herramientas importantes para la toma de decisiones enfocadas a eliminar o disminuir la incidencia de las fallos sus efectos y consecuencias (Escobar et al., 2003), además es útil en múltiples aplicaciones como: predecir la fiabilidad de un activo nuevo, mejorar la calidad de su diseño, medir la fiabilidad en operación, permitiendo determinar la longevidad y la repercusión de los fallos en los sistemas o equipos industriales (Nachias, 1995). El estudio de la fiabilidad se basa en la investigación de los tiempos operativos hasta el fallo. 
Se reconoce que los componentes de activos industriales tienen ciclos de vida finitos, en la práctica se distinguen dos tipos de componentes, los que son reparados y los que son reemplazados después de la ocurrencia del fallo. Con esta premisa se distinguen dos tiempos, el tiempo hasta el fallo utilizado desde que el dispositivo empieza a funcionar hasta el fallo donde es reemplazado comentado en Nachias (1995) y Crespo et al. (2004), el tiempo entre fallos que es el tiempo operativo entre dos fallos consecutivos (Comité Europeo de Normalización, 2018). De los tiempos mencionados derivan otros conceptos como MTBF (siglas en ingles de Tiempo Medio Entre Fallos) y MTTF (Tiempo Medio para el Fallo) (Gómez, 2002).

La fiabilidad está definida como la capacidad de un activo para cumplir óptimamente con las funciones para lo que fue adquirido, analizado en un período de tiempo y bajo condiciones operacionales definidas (Holmber et al., 1991; Mors, 2009).

El inicio para el estudio de la fiabilidad en equipos no reparables, los cuales son reemplazados después de la ocurrencia de un fallo está enfocado en el estudio de la variable aleatoria tiempos de funcionamiento correcto hasta el fallo, para luego analizar su contraparte la causa del estado de fallo.

El indicador más básico para el estudio de la fiabilidad en equipos no reparable es MTTF (Tiempo Medio Para la Falla), como se visualiza en la figura 1. "Definido como la suma de los tiempos operativos hasta el fallo dividido para el número de fallos en un tiempo de análisis" (Ruiz, 2013).

$\mathrm{MTTF}=\sum \mathrm{TTF} /$ Numero de fallos (1)

\section{Figura 1}

Tiempo medio hasta el fallo

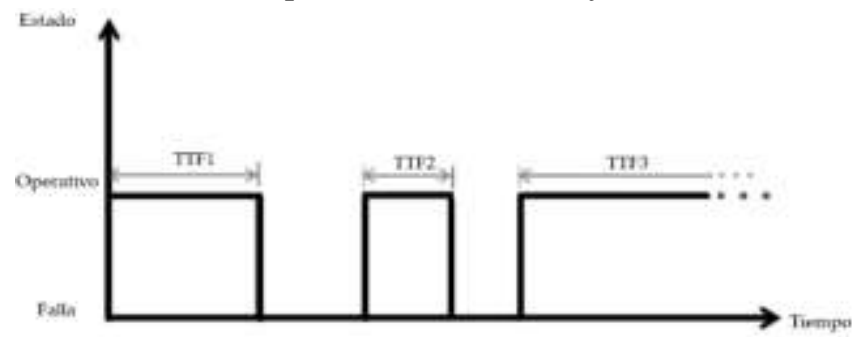

En un activo no reparable el tiempo para la falla trata de determinar la probabilidad que un activo trabaje sin fallas en un tiempo igual o superior al tiempo de análisis:

$$
\text { Fiabilidad }(\mathrm{t})=\operatorname{Pr}(\mathrm{t} \geq \text { tiempo de análisis })
$$

Las funciones de distribución más utilizadas para el estudio de la fiabilidad:" la distribución de densidad de probabilidad $f(t)$, función de densidad acumulada $F(t)$ y 
función acumulada inversa o de Supervivencia $\mathrm{R}(\mathrm{t})$ de la variable aleatoria continua tiempo hasta la falla información compartida por (Parra \& Crespo, 2014), ver figura 2.

\section{Figura 2}

Distribuciones de probabilidad del tiempo hasta el fallo
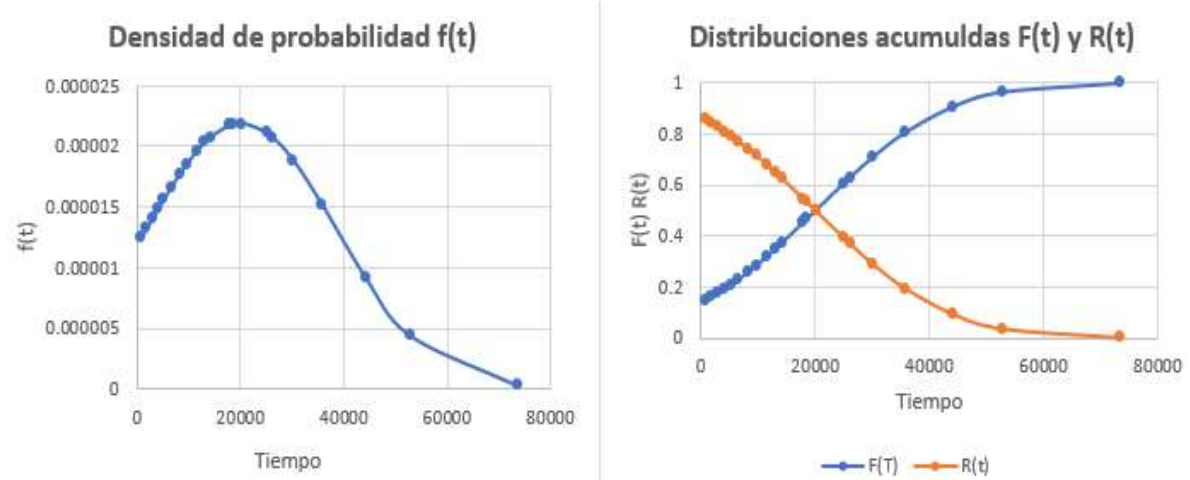

La fiabilidad se define:

$\mathrm{R}(\mathrm{t})=\operatorname{Pr}(\mathrm{t} \geq$ tiempo de análisis (ta) $)=\int_{t a}^{\infty} f(t) d t$

Donde ta es el tiempo de análisis.

La ecuación anterior define a la fiabilidad $\mathrm{R}(\mathrm{t})$ como la probabilidad de que el activo no falle después del tiempo definido para el análisis (Parra \& Crespo, 2016), y se calcula como la integral definida desde el tiempo de análisis hasta el infinito de la función de densidad de probabilidad.

Como la probabilidad de fallo más la probabilidad de supervivencia es igual a 1 (Acuña, 2003) se puede definir que:

$\mathrm{R}(\mathrm{t})=1-\operatorname{Pr}(\mathrm{t} \geq \mathrm{ta})=1-\int_{t a}^{\infty} f(t) d t=1-\mathrm{F}(\mathrm{t})$

El tiempo medio para fallar MTTF es el parámetro utilizado para el diseño y selección de sistemas y se calcula como la media de la distribución de densidad acumulada del tiempo para la falla:

$\mathrm{MTTF}=\int_{0}^{\infty} t f(t) d t=\int_{0}^{\infty} R(t) d t$

Su Representación gráfica se muestra en la Figura 3. 


\section{Figura 3}

Representación del tiempo para la falla

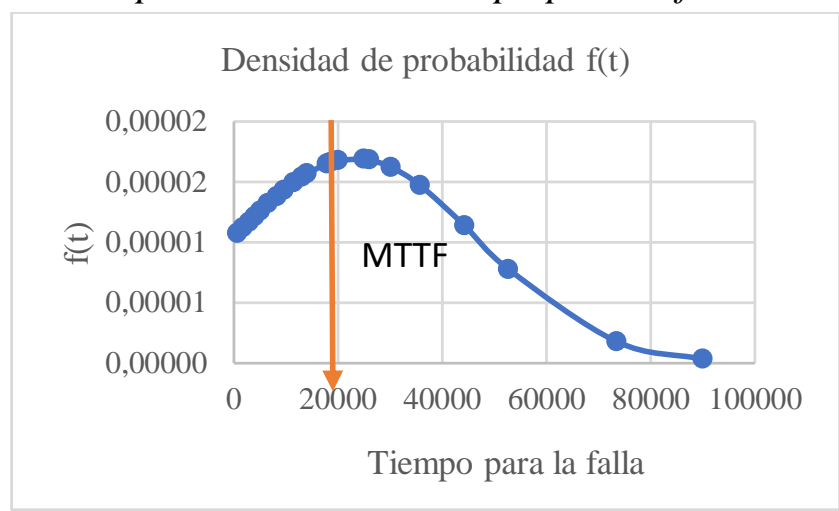

Otra representación del tiempo para la falla en la función de la tasa de fallos, la media de la tasa de fallos es el comportamiento del número de fallas por unidad de tiempo mientras que la tasa de fallos instantánea llamada también como "la función del riesgo y se describe como probabilidad de que un equipo que ha sobrevivido a un tiempo t sobreviva en un intervalo mayor a este tiempo $(\mathrm{t}+\Delta \mathrm{t}$ )" (Yánez et al., 2004). Se expresa como:

$\lambda(\mathrm{t})=\frac{f(t)}{R(t)}=\frac{f(t)}{1-F(t)}$

Los tiempos hasta el fallo son variables aleatorias continuas, estos datos se representan por medio de distribuciones, lo importante es determinar cuál de las distribuciones proporciona un mejor ajuste al conjunto de datos.

Entre las distribuciones más comunes para el estudio de la fiabilidad con variables aleatorias continuas son expresa (Yánez et al., 2004)

- Distribución de Weibull

- Distribución Gamma

- Distribución Normal

- Distribución Log - Normal

- Distribución Exponencial

Las expresiones para el cálculo de las funciones de distribución tomadas de Ebeling (1997) y Knezevic (1996), se describen en la tabla 1. 


\section{Tabla 1}

Funciones distribuciones continuas

\begin{tabular}{|c|c|c|c|c|}
\hline FUNCIÓN & Densidad $f(t)$ & Distribución F(t) & Supervivencia $\mathrm{R}(\mathrm{t})$ & $\begin{array}{c}\text { Riesgo } \\
\lambda(t)\end{array}$ \\
\hline $\begin{array}{l}\text { Exponenci } \\
\text { al }\end{array}$ & $\lambda e^{-\lambda t}$ & $1-e^{-\lambda t}$ & $e^{-\lambda t}$ & $\lambda$ \\
\hline Weibull & $\frac{\beta}{\alpha}\left(\frac{t-\Upsilon}{\alpha}\right)^{\beta-1} e^{-\left(\frac{t-\Upsilon}{\alpha}\right)^{\beta}}$ & $1-e^{-\left(\frac{t-\gamma}{\alpha}\right)^{\beta}}$ & $e^{-\left(\frac{t-\Upsilon}{\alpha}\right)^{\beta}}$ & $\frac{\beta}{\alpha}\left(\frac{t-\Upsilon}{\alpha}\right)^{\beta-1}$ \\
\hline Normal & $\frac{1}{6 \sqrt{2 \Pi}} e^{-\frac{1}{2}\left(\frac{t-\mu}{6}\right)^{2}}$ & $\int_{-\infty}^{t} \frac{1}{6 \sqrt{2}} e^{-\frac{1}{2}\left(\frac{t-\mu}{6}\right)^{2} d(t)}$ & $\int_{t}^{\infty} \frac{1}{6 \sqrt{2 \Pi}} e^{-\frac{1}{2}\left(\frac{t-\mu}{6}\right)^{2} d(t)}$ & $\frac{f(t)}{R(t)}$ \\
\hline $\begin{array}{l}\text { Log- } \\
\text { Normal }\end{array}$ & $\frac{1}{6 \sqrt{2 \Pi}} e^{-\frac{1}{2}\left(\frac{\ln (t)-\mu}{6}\right)^{2}}$ & $\int_{-\infty}^{t} \frac{1}{6 \sqrt{2 \Pi}} e^{-\frac{1}{2}\left(\frac{t-\mu}{6}\right)^{2} d(t}$ & $\int_{t}^{\infty} \frac{1}{6 \sqrt{2 \Pi}} e^{-\frac{1}{2}\left(\frac{t-\mu}{6}\right)^{2} d(t)}$ & $\frac{f(t)}{R(t)}$ \\
\hline Gamma & $\frac{1}{\beta^{\alpha} \Gamma(\alpha)} t^{\alpha-1} e^{-\frac{t}{\beta}}$ & $\frac{1}{\beta^{\alpha} \Gamma(\alpha)} \int_{0}^{t} t^{\alpha-1} e^{-\frac{t}{\beta}} d($ & $\frac{1}{\beta^{\alpha} \Gamma^{\prime}(\alpha)} \int_{t}^{t \infty} t^{\alpha-1} e^{-\frac{t}{\beta}} d(t$ & $\frac{f(t)}{R(t)}$ \\
\hline
\end{tabular}

Los parámetros para el cálculo de cada distribución de los puede apreciar en la tabla 2.

Tabla 2

Ecuaciones de parámetros

\begin{tabular}{|c|c|c|}
\hline FUNCIÓN & & PARÁMETROS \\
\hline Exponencial & $\lambda=\frac{n}{\sum_{i=1}^{n} t i}$ & - \\
\hline Weibull & $\alpha=\frac{\left(\sum_{t=1}^{n} t i^{\beta}\right)^{1 / \beta}}{n}$ & $\frac{\sum_{i=1}^{n}\left[X i^{t i} \ln (t i)\right]}{n^{2} \sum_{t=i}^{n} t i \beta}-\frac{1}{\beta}=\frac{1}{n} \sum_{t=i}^{n} \ln (t i)$ \\
\hline Normal & $\mu=\frac{\sum_{t=i}^{n} t i}{n}$ & $6^{2}=\frac{1}{(n-1)} \sum_{t=i}^{n}(t i-\mu)^{2}$ \\
\hline Log - Normal & $\mu \mathrm{t}=\frac{\sum_{i=1}^{n} \ln (t i)}{n}$ & $6 t=\frac{1}{n} \sum_{i=1}^{n}(\ln (t i)-\mu t)^{2}$ \\
\hline Gamma & $\alpha=\frac{(n-1)\left(\sum_{i=1}^{n} t i\right)^{2}}{n^{2} \sum_{i=1}^{n}(t i-u x)^{2}}$ & $\beta=\frac{n \sum_{i=1}^{n}(t i-u x)^{2}}{(n-1) \sum_{t=i}^{n} t i}$ \\
\hline
\end{tabular}


"Seleccionadas las distribuciones hipotéticas a evaluar en función de la variable aleatoria se debe realizar una prueba de bondad ajuste" (Gasca et al., 2017), para determinar cuál de las distribuciones hace un mejor ajuste al grupo de datos, hay varias pruebas de bondad de ajuste de bondad:

- Prueba Chi Cuadrado $x^{2}$ (Utilizada en variables aleatorias discretas)

- Prueba de Kolmogorov - Smirnov

- Prueba de Anderson - Darling

"La prueba de Kolmogorov - Smirnov es la más utilizada" (Yánez et al., 2004). los pasos a seguir son los siguientes:

Calcular la función acumulada $\mathrm{F}(\mathrm{t})$ para cada una de las distribuciones seleccionadas y calcular la función de distribución empírica $\mathrm{F}_{\mathrm{e}}(\mathrm{t})$ :

$$
\mathrm{F}_{\mathrm{e}}(\mathrm{t})=\frac{n}{N}
$$

Donde $\mathrm{n}$ es el número de orden y $\mathrm{N}$ el tamaño de la muestra

1. Graficar las distribuciones

2. Calcular el K-S valor máximo, utilizando:

$$
\mathrm{K}-\mathrm{S}_{\text {VALOR }}=\left(\left|\mathrm{F}(\mathrm{t})-\mathrm{F}_{\mathrm{e}}(\mathrm{t})\right|\right) ;\left(\left|\mathrm{F}(\mathrm{t})-\mathrm{F}_{\mathrm{e}}(\mathrm{t}-1)\right|\right)
$$

3. Comprar el K-S $\mathrm{VALOR}$ con valor el correspondiente de la tabla del Kolmogorov Smirnov seleccionado con el número de datos y con un valor de significancia del $5 \%$. Si el dato es menor que el de la tabla la hipótesis es verdadera y la distribución es seleccionada, si varias distribuciones cumplen el requerimiento se selecciona la de menor K-SVALOR, en la tabla 3 corresponde a los valores críticos de la prueba del Kolmogorov - Smirnov.

Tabla 3

Valores críticos. Test de Kolmogorov-Smirnov

\begin{tabular}{cccccc}
\hline & \multicolumn{5}{c}{$\boldsymbol{r}$} \\
\cline { 2 - 6 } & 0.20 & 0.15 & 0.10 & 0.05 & 0.01 \\
\hline 1 & 0.900 & 0.925 & 0.950 & 0.975 & 0.995 \\
2 & 0.684 & 0.726 & 0.776 & 0.842 & 0.929 \\
3 & 0.565 & 0.597 & 0.642 & 0.708 & 0.828 \\
4 & 0.494 & 0.525 & 0.564 & 0.624 & 0.733 \\
5 & 0.446 & 0.474 & 0.510 & 0.565 & 0.669 \\
6 & 0.410 & 0.436 & 0.470 & 0.521 & 0.618 \\
7 & 0.381 & 0.405 & 0.380 & 0.486 & 0.577 \\
8 & 0.358 & 0.381 & 0.411 & 0.457 & 0.543 \\
9 & 0.339 & 0.360 & 0.388 & 0.432 & 0.514 \\
10 & 0.322 & 0.342 & 0.368 & 0.410 & 0.490 \\
11 & 0.307 & 0.326 & 0.352 & 0.391 & 0.468 \\
\hline
\end{tabular}




\begin{tabular}{|c|c|c|c|c|c|}
\hline \multirow{2}{*}{$n$} & \multicolumn{5}{|c|}{$\mathbf{r}$} \\
\hline & 0.20 & 0.15 & 0.10 & 0.05 & 0.01 \\
\hline 12 & 0.295 & 0.313 & 0.338 & 0.375 & 0.450 \\
\hline 13 & 0.284 & 0.302 & 0.325 & 0.361 & 0.433 \\
\hline 14 & 0.274 & 0.292 & 0.314 & 0.349 & 0.418 \\
\hline 15 & 0.266 & 0.283 & 0.304 & 0.338 & 0.404 \\
\hline 16 & 0.258 & 0.274 & 0.295 & 0.328 & 0.392 \\
\hline 17 & 0.250 & 0.266 & 0.286 & 0.318 & 0.381 \\
\hline 18 & 0.244 & 0.259 & 0.278 & 0.309 & 0.371 \\
\hline 19 & 0.237 & 0.252 & 0.272 & 0.301 & 0.363 \\
\hline 20 & 0.231 & 0.246 & 0.264 & 0.294 & 0.356 \\
\hline 25 & 0.210 & 0.220 & 0.240 & 0.270 & 0.320 \\
\hline 30 & 0.190 & 0.200 & 0.220 & 0.240 & 0.290 \\
\hline 35 & 0.180 & 0.190 & 0.210 & 0.230 & 0.270 \\
\hline$>35$ & $1.07 / \sqrt{ } \mathrm{n}$ & $1.14 / \sqrt{ } \mathrm{n}$ & $1.22 / \sqrt{ } \mathrm{n}$ & $1.36 / \sqrt{ } \mathrm{n}$ & $1.63 / \sqrt{ } \mathrm{n}$ \\
\hline
\end{tabular}

\section{Metodología}

Recolectados los tiempos hasta el fallo (ti) de los elementos no reparables, se deben plantear tantas hipótesis como distribuciones de hayan seleccionado (la hipótesis está relacionada con que los datos recolectados se ajusten a la distribución analizada).

Para cada distribución calcular sus respectivos parámetros utilizando las ecuaciones específicas, ver tabla 2.

Para cada valor de ti (tiempo hasta el fallo) calcular la probabilidad de falla acumulada teórica $\mathrm{F}(\mathrm{t})$.

Luego debe calcularse la probabilidad de falla empírica $\mathrm{F}_{\mathrm{e}}$ (ecuación 5) para cada valor de ti.

Graficar cada probabilidad de falla teórica Vs la empírica con todas las distribuciones analizadas, figura 4.

Aplicar la prueba de bondad de ajuste de Kolmogorov - Smirnov para ello calcular el KSVALOR de cada distribución y compáralo con el valor crítico del test de KolmogorovSmirnov (VKS). Si el KS-VALOR es inferior al valor VKS. la hipótesis no es rechazada y podría ser seleccionada para el mejor ajuste. El proceso anterior se repite en todas las distribuciones, de las hipótesis no rechazadas se debe seleccionar la de menor valor y así proseguir con las estimaciones de la fiabilidad. 


\section{Figura 4}

Representación $F(t)$ vs $F_{e}(t)$

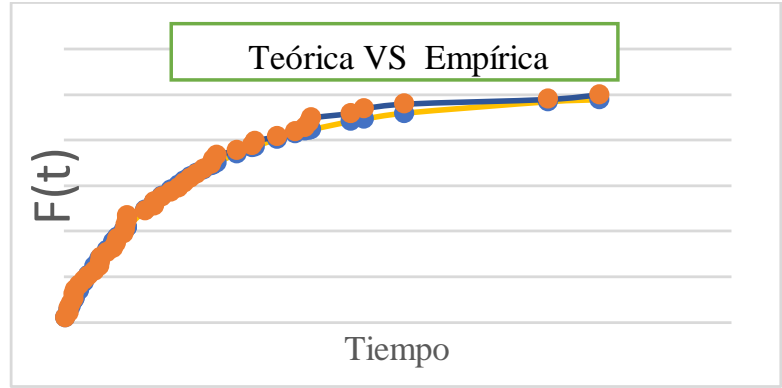

\section{Resultados}

Para el análisis se tomaron como muestra los fallos de 250 lámparas de $\mathrm{Na}$ (Sodio) de alta presión de 100W, con boquilla e40, pertenecientes al alumbrado público. En análisis se enfocó únicamente en las luminarias. El tiempo de recolección de las fallas se lo realizó en un período de 26 semanas, la información fue tomada de las órdenes de mantenimiento correctivo de la empresa, en la tabla 4 representa una muestra de la recolección de datos.

\section{Tabla 4}

Muestra de datos de falla

\begin{tabular}{cccc}
\hline $\mathrm{n}$ & Falla & Fecha y hora de la falla & Horas de uso acumulado \\
\hline 1 & Falla de luminaria & $2020-06-16$ 19:00:00 & 13305.5 \\
2 & Falla de luminaria & $2020-06-1600: 01: 00$ & 13296 \\
3 & Falla de luminaria & $2020-06-1311: 00: 00$ & 13265.5 \\
4 & Falla de luminaria & $2020-06-1214: 15: 00$ & 13255 \\
5 & Falla de luminaria & $2020-06-1200: 30: 00$ & 13248 \\
& & $\ldots$ & $\ldots$ \\
$\ldots$ & $\ldots$ & $\ldots$ & $\ldots$ \\
$\ldots$ & $\ldots$ & $2020-01-0308: 30: 00$ & 55.5 \\
202 & Falla de luminaria & $2020-01-0308: 10: 00$ & 55.5 \\
203 & Falla de luminaria & $2020-01-0211: 15: 00$ & 45 \\
204 & Falla de luminaria & $2020-01-0212: 15: 00$ & 45.5 \\
205 & Falla de luminaria & & \\
\hline
\end{tabular}

Una muestra del cálculo de la función acumulada empírica (ecuación 5) se presenta en la tabla 5 .

$\mathrm{F}_{\mathrm{e}}(\mathrm{t})=\frac{n}{N}$, donde $\mathrm{n}$ es el número de orden y $\mathrm{N}$ el tamaño de la muestra. 


\section{Tabla 5}

Muestra del cálculo de la función de probabilidad empírica

\begin{tabular}{ccc}
\hline $\mathrm{n}$ & $\begin{array}{c}\text { Tiempo acumulado } \\
\text { hasta el fallo }(\mathrm{t})\end{array}$ & $\mathrm{Fe}(\mathrm{t})$ \\
\hline 1 & 19.011537 & 0.004 \\
2 & 37.559079 & 0.008 \\
3 & 40.322394 & 0.012 \\
4 & 49.451985 & 0.016 \\
-- & -- & -- \\
-- & -- & -- \\
247 & 321.074163 & 0.988 \\
248 & 321.81291 & 0.992 \\
249 & 323.771535 & 0.996 \\
250 & 337.06206 & 1 \\
\hline
\end{tabular}

Para cada tiempo se calcula la función de probabilidad de fallo acumulada $F(t)$ para cada una de las distribuciones seleccionadas y se grafica la distribución versus la empírica. Figuras 5,6,7,8,9

Distribución Exponencial. $\quad F(t)=1-\mathrm{e}^{-\lambda t}$

$\lambda=$ Tasa de fallos; $\mathrm{t}$ TTF $=$ Tiempo hasta el fallo

\section{Figura 5}

$F(t)$ Exponencial vs $F_{e}(t)$

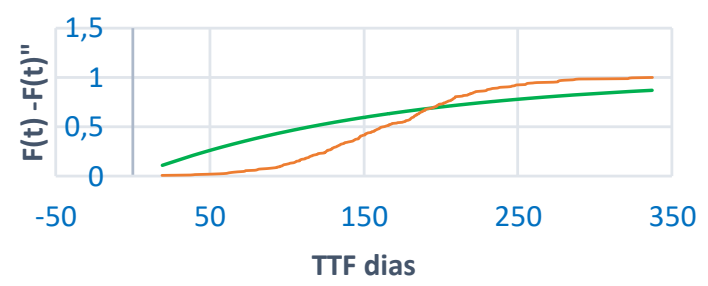

$-F(t)$ exponencial — $F(t)$ empírica

Distribución de Weibull.

$$
\mathbf{F}(\mathrm{t})=1-e^{-[t / \alpha]^{\beta}}
$$

$\beta=$ Parámetro de forma $\alpha=$ Parámetro de escala 
Figura 6

$F(t)$ Weibull vs $F_{e}(t)$

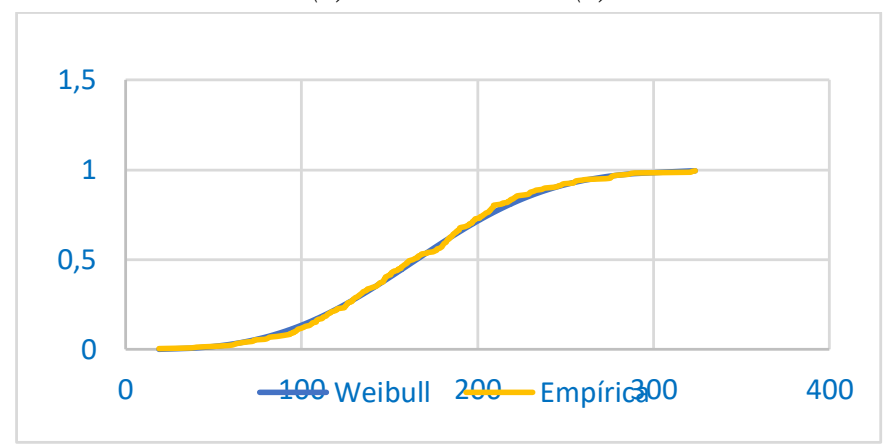

Distribución Normal.

$$
F(t)=\int_{-\infty}^{t} \frac{1}{6 \sqrt{2 \prod}} e^{-\frac{1}{2}\left(\frac{t-\mu}{6}\right)^{2} d(t)}
$$

$\mu=$ Media; $\sigma=$ Desviación estándar

\section{Figura 7}

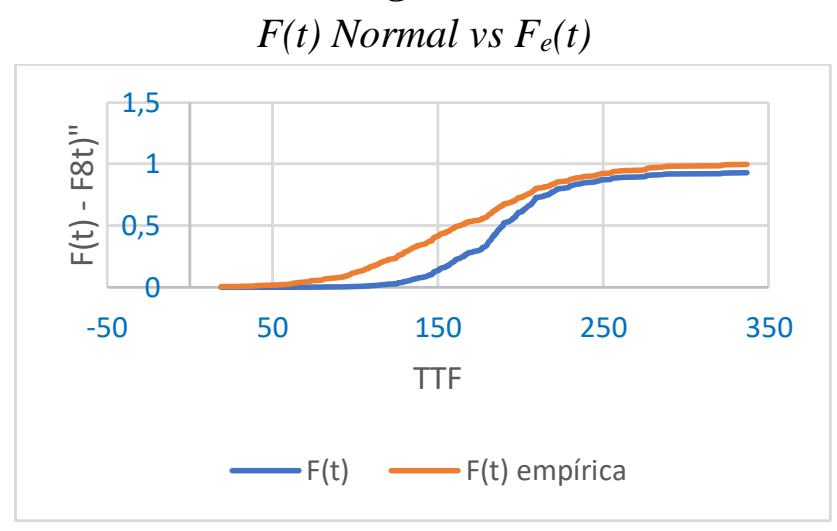

Distribución Log-Normal.

$$
\int_{-\infty}^{t} \frac{1}{6 \sqrt{2 \prod}} e^{-\frac{1}{2}\left(\frac{t-\mu}{6}\right)^{2} d(t)}
$$

$\mu=$ Media $; \sigma=$ Desviación estándar 


\section{Figura 8}

$F(t) \log$-Normal vs $F_{e}(t)$

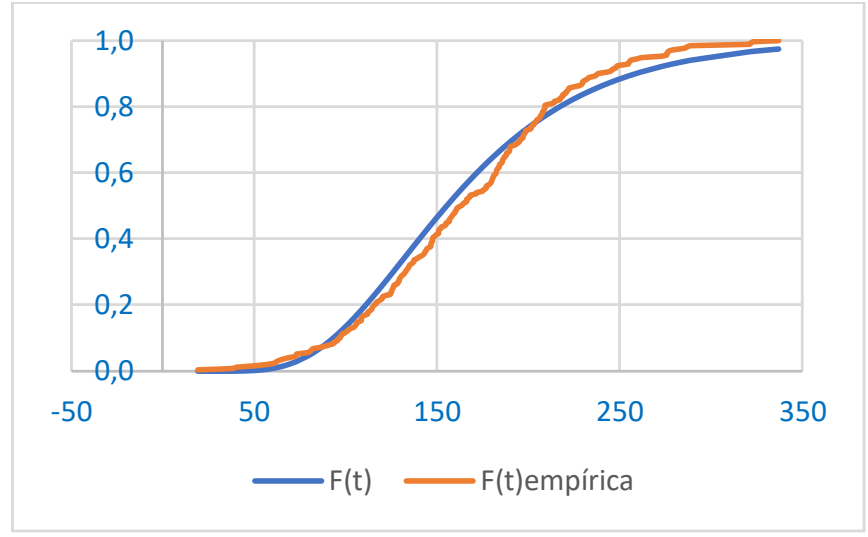

Log-Normal.

$$
\frac{1}{\beta^{\alpha} \tilde{\Gamma}(\alpha)} \int_{0}^{t} t^{\alpha-1} e^{-\frac{t}{\beta}} d(t)
$$

$\beta=$ Parámetro de forma; $\alpha=$ Parámetro de escala

\section{Figura 9}

$F(t)$ Gamma vs $F_{e}(t)$

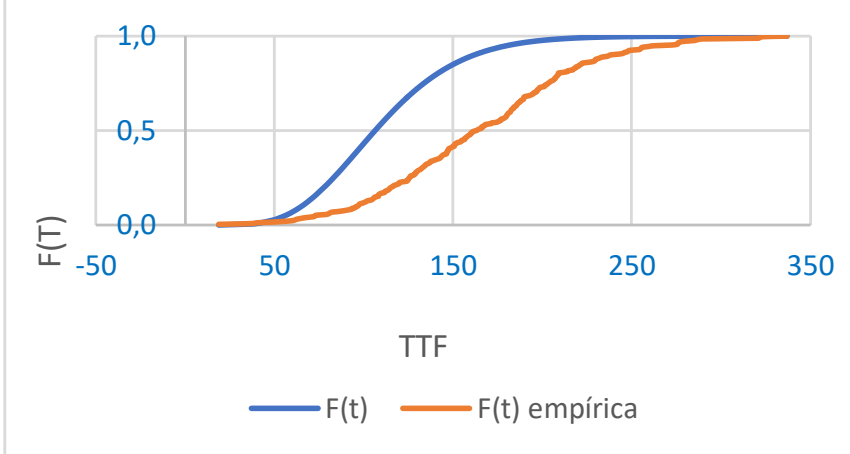

Gráficamente la curvas con mejor ajuste es la Distribución de Weibull, lo cual se confirmará con la prueba de Kolmogorov-Smirnov. Se debe calcular la función de fallos acumulada para cada distribución, como se muestra en la tabla 6 .

\section{Tabla 6}

Muestra del cálculo de las funciones de fallo para las distribuciones

\begin{tabular}{cccccccc}
\hline $\begin{array}{c}\text { Falla No } \\
\text { "i" }\end{array}$ & $\begin{array}{c}\mathrm{t} \text { "i" } \\
\text { (días) }\end{array}$ & $\begin{array}{c}\mathrm{F}(\mathrm{t}) \\
\text { EXPONENCIAL }\end{array}$ & $\begin{array}{c}\mathrm{F}(\mathrm{t}) \\
\text { WEIBULL }\end{array}$ & $\begin{array}{c}\mathrm{F}(\mathrm{t}) \\
\text { NORMAL }\end{array}$ & $\begin{array}{c}\mathrm{F}(\mathrm{t}) \text { Log- } \\
\text { NORMAL }\end{array}$ & GAMMA & $\begin{array}{c}\mathrm{F}(\mathrm{t}) \\
\text { EMPÍRICA }\end{array}$ \\
\hline 1 & 19 & 0.108 & 0.0008 & 0.002 & 0 & 0.0001 & 0.004 \\
2 & 37.6 & 0.202 & 0.0068 & 0.0021 & 0.00019 & 0.0056 & 0.008 \\
\hline
\end{tabular}




\section{Tabla 6}

Muestra del cálculo de las funciones de fallo para las distribuciones (continuación)

\begin{tabular}{cccccccc}
\hline $\begin{array}{c}\text { Falla No } \\
\text { "i" }\end{array}$ & $\begin{array}{c}\mathrm{t} \text { "i" } \\
\text { (días) }\end{array}$ & $\begin{array}{c}\text { EXPONENCIA } \\
\text { L }\end{array}$ & $\begin{array}{c}\mathrm{F}(\mathrm{t}) \\
\text { WEIBULL }\end{array}$ & $\begin{array}{c}\mathrm{F}(\mathrm{t}) \\
\text { NORMAL }\end{array}$ & $\begin{array}{c}\mathrm{F}(\mathrm{t}) \text { Log- } \\
\text { NORMAL }\end{array}$ & $\begin{array}{c}\text { GAM } \\
\text { MA }\end{array}$ & $\begin{array}{c}\mathrm{F}(\mathrm{t}) \\
\text { EMPÍRICA }\end{array}$ \\
\hline 3 & 40.3 & 0.215 & 0.0085 & 0.0022 & 0.00037 & 0.0085 & 0.012 \\
4 & 49.5 & 0.257 & 0.016 & 0.0024 & 0.0021 & 0.0262 & 0.016 \\
5 & 56.3 & 0.287 & 0.0239 & 0.0025 & 0.00557 & 0.0502 & 0.02 \\
-- & -- & -- & -- & -- & -- & -- & -- \\
-- & -- & -- & -- & -- & -- & -- & - \\
247 & 321.1 & 0.855 & 0.996 & 0.919 & 0.965 & 1 & 0.988 \\
248 & 321.8 & 0.855 & 0.996 & 0.922 & 0.966 & 1 & 0.992 \\
249 & 323.8 & 0.857 & 0.996 & 0.924 & 0.967 & 1 & 0.996 \\
250 & 337.1 & 0.868 & 0.998 & 0.927 & 0.974 & 1 & 1 \\
\hline
\end{tabular}

Se procede a calcular los valores absolutos de las diferencias entre valores de las probabilidades acumuladas teóricas y empíricas, para todos los datos de la muestra, como se indica en las siguientes ecuaciones:

- $\left|\mathrm{F}\left(\mathrm{t}_{\mathrm{i}}\right)-\dot{\mathrm{F} e}\left(\mathrm{t}_{\mathrm{i}}\right)\right|$

- $\left|\mathrm{F}\left(\mathrm{t}_{\mathrm{i}}\right)-\dot{\mathrm{F} e}\left(\mathrm{t}_{\mathrm{i}}-1\right)\right|$

La tabla 7 es una muestra del cálculo de los parámetros para la prueba de ajuste de bondad K-S. Una vez calculados los valores se debe identificar los valores más altos para cada una de las distribuciones, estos valores se deben comparar con los valores críticos para el test de Kolmogorov-Smirnov de la tabla 3. Para el estudio se tomó un nivel de significancia del 5\% y como la muestra es de 250 lámparas se debe calcular con la expresión:

Valor Crítico para 5\% de significancia $=\frac{1.36}{\sqrt{250}}=0.086$

\section{Tabla 7}

Muestra del cálculo de los parámetros de la prueba de ajuste de bondad de Kolmogorov - Smirnov

\begin{tabular}{ccccc}
\hline Falla No " $i "$ & $F(t)$ EXPONENCIAL & $F(t)$ EMPIRICA & $\left|\mathrm{F}\left(\mathrm{t}_{\mathrm{i}}\right)-\dot{\mathrm{F}}\left(\mathrm{t}_{\mathrm{i}}\right)\right|$ & $\left|\mathrm{F}\left(\mathrm{t}_{\mathrm{i}}\right)-\dot{\mathrm{F}}\left(\mathrm{t}_{\mathrm{i}}-1\right)\right|$ \\
1 & 0.108 & 0.004 & 0.104 & 0.198 \\
2 & 0.202 & 0.008 & 0.194 & 0.207 \\
3 & 0.215 & 0.012 & 0.203 & 0.245 \\
-- & -- & -- & -- & -- \\
\hline Falla No " $i$ " & $F(t)$ WEIBULL & $F(t)$ EMPÍRICA & $\left|\mathrm{F}\left(\mathrm{t}_{\mathrm{i}}\right)-\dot{\mathrm{F}}\left(\mathrm{t}_{\mathrm{i}}\right)\right|$ & $\left|\mathrm{F}\left(\mathrm{t}_{\mathrm{i}}\right)-\dot{\mathrm{F}}\left(\mathrm{t}_{\mathrm{i}}-1\right)\right|$ \\
1 & 0.0008 & 0.004 & 0.003 & 0.003 \\
2 & 0.0068 & 0.008 & 0.001 & 0.001 \\
3 & 0.0085 & 0.012 & 0.003 & 0.004 \\
-- & -- & -- & -- & --
\end{tabular}




\section{Tabla 7}

Muestra del cálculo de los parámetros de la prueba de ajuste de bondad de Kolmogorov - Smirnov (continuación)

\begin{tabular}{ccccc}
\hline Falla No "i" & $F(t)$ NORMAL & $F(t)$ EMPÍRICA & $\left|\mathrm{F}\left(\mathrm{t}_{\mathrm{i}}\right)-\dot{\mathrm{F}}\left(\mathrm{t}_{\mathrm{i}}\right)\right|$ & $\left|\mathrm{F}\left(\mathrm{t}_{\mathrm{i}}\right)-\dot{\mathrm{F}}\left(\mathrm{t}_{\mathrm{i}}-1\right)\right|$ \\
1 & 0.0020 & 0.004 & 0.002 & 0.002 \\
2 & 0.0021 & 0.008 & 0.006 & 0.006 \\
3 & 0.0022 & 0.012 & 0.010 & 0.010 \\
-- & -- & -- & -- & -- \\
\hline Falla No $^{\prime \prime} i^{\prime \prime}$ & $F(t)$ Log- NORMAL & $F(t)$ EMPIRICA & $\left|\mathrm{F}\left(\mathrm{t}_{\mathrm{i}}\right)-\dot{\mathrm{F}}\left(\mathrm{t}_{\mathrm{i}}\right)\right|$ & $\left|\mathrm{F}\left(\mathrm{t}_{\mathrm{i}}\right)-\dot{\mathrm{F}}\left(\mathrm{t}_{\mathrm{i}}-1\right)\right|$ \\
1 & 0.0000001 & 0.004 & 0.004 & 0.004 \\
2 & 0.0001920 & 0.008 & 0.008 & 0.008 \\
3 & 0.0003713 & 0.012 & 0.012 & 0.010 \\
-- & -- & -- & -- & -- \\
\hline Falla No ${ }^{\prime \prime}$ & $F(t)$ GAMMA & $F(t)$ EMPIRICA & $\left|\mathrm{F}\left(\mathrm{t}_{\mathrm{i}}\right)-\dot{\mathrm{F}}\left(\mathrm{t}_{\mathrm{i}}\right)\right|$ & $\left|\mathrm{F}\left(\mathrm{t}_{\mathrm{i}}\right)-\dot{\mathrm{F}}\left(\mathrm{t}_{\mathrm{i}}-1\right)\right|$ \\
1 & 0.000063 & 0.004 & 0.004 & 0.002 \\
2 & 0.005636 & 0.008 & 0.002 & 0.001 \\
3 & 0.008539 & 0.012 & 0.003 & 0.014 \\
-- & -- & -- & -- & -
\end{tabular}

Los valores máximos obtenidos para las distribuciones se muestran en la tabla 8 .

Tabla 8

Valores máximos de los parámetros de la prueba de

\begin{tabular}{ll}
\multicolumn{2}{c}{ bondad de ajuste } \\
\hline Exponencial & 0.350 \\
Weibull & 0.039 \\
\hline Normal & 0.278 \\
\hline Log-normal & 0.079 \\
\hline Gamma & 0.460 \\
\hline
\end{tabular}

Estos valores se comparan con el valor crítico de la prueba K-S (0.086), como se puede apreciar dos distribuciones están bajo este valor Weibull y Log-Normal, el resto de las hipótesis son rechazadas, Como el valor de la distribución de Weibull es menor que el de la distribución Log-Normal se escoge esta distribución para continuar con el análisis confirmando de esta manera los resultados obtenidos en el análisis gráfico.

Con la distribución de Weibull se construyen Las curvas de Supervivencia R(t), La de Probabilidad de Fallo F(t), La función de la densidad $\mathrm{f}(\mathrm{t})$ y la Función del Riesgo $\lambda(\mathrm{t})$ para continuar el estudio de las luminarias figura 10. 


\section{Figura 10}

Funciones de la distribución de Weibull

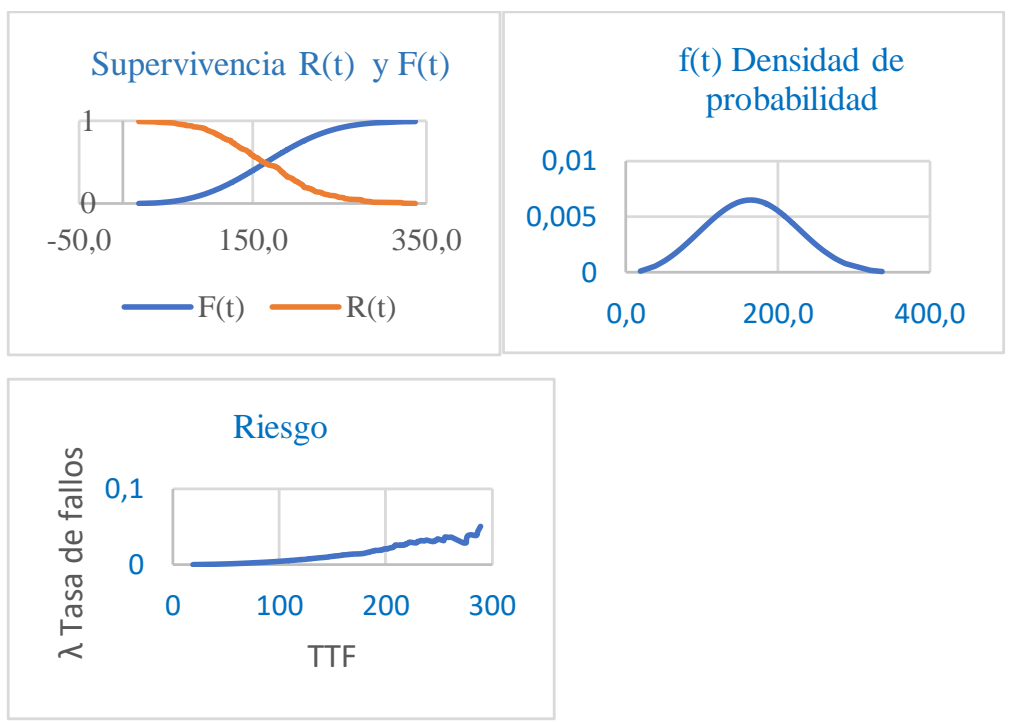

Se realizó una corrida de datos para varios tiempos de ensayo en días para calcular la probabilidad de funcionamiento y fallas para las lámparas de sodio obteniendo los resultados de la tabla 9 donde se verifica las variaciones de las probabilidades fallo y supervivencia en el tiempo

\section{Tabla 9}

Probabilidad de fallo y supervivencia

\begin{tabular}{ccc}
\hline \multicolumn{3}{c}{ Probabilidad de fallo y supervivencia } \\
\hline $\mathrm{t}$ (días) & $\begin{array}{c}\mathrm{F}(\mathrm{t}) \text { Probabilidad de } \\
\text { fallo }\end{array}$ & $\begin{array}{c}\mathrm{R}(\mathrm{t}) \text { Probabilidad de } \\
\text { supervivencia }\end{array}$ \\
\hline 10 & $0 \%$ & $100 \%$ \\
50 & $2 \%$ & $98 \%$ \\
100 & $13 \%$ & $87 \%$ \\
200 & $72 \%$ & $28 \%$ \\
350 & $100 \%$ & $0 \%$ \\
\hline
\end{tabular}

Un detalle de la variación de la probabilidad de fallo en el tiempo se lo representa en la figura 11 
Figura 11

Variación de la Probabilidad de fallo y Supervivencia en el tiempo

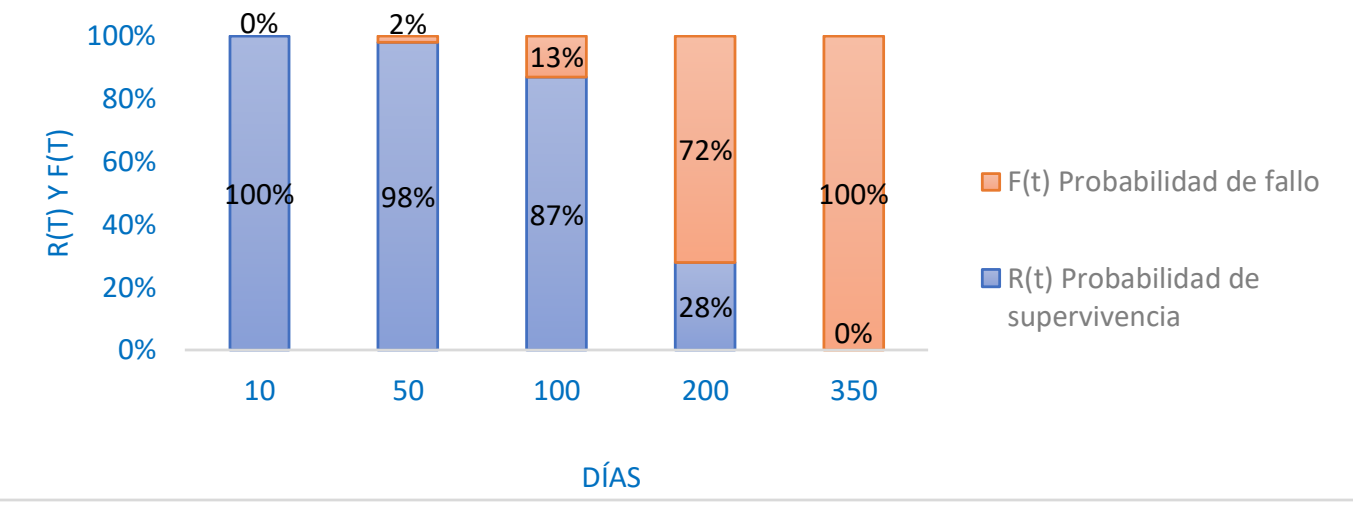

\section{Conclusiones}

- El análisis de la fiabilidad para equipos reparable se los puede enfocar en forma diferente, puede utilizarse para fallas en equipos similares montados en máquinas diferentes como las flotas o luminarias como es el caso de estudio, también se lo puede hacer para un solo equipo siempre que se tenga un listado de fallos lo suficientemente extenso para realizar los cálculos estadísticos, se recomienda cinco datos como mínimo.

- Si los datos no se ajustan a ninguna distribución paramétrica se pueda trabajar con la distribución no paramétrica o empírica

- El propósito de los cálculos de la fiabilidad o supervivencia $\mathrm{R}(\mathrm{t})$ están destinados a predecir la probabilidad que un equipo siga funcionando correctamente en un período d tiempo de análisis. Pero la idea será siempre mejorar estos indicadores para esto se deberán plantear estrategias para que esto ocurra

- Estos cálculos se los puede plantear por marca para determinar la más fiable, combinando este indicador con un análisis costo beneficio será de mucha utilidad en la selección de equipos.

\section{Referencias Bibliográficas}

Acuña, J. (2003). Ingeniería de la Confiabilidad. Costa Rica: Tecnológica de Costa Rica.

Comité Europeo de Normalización. (2018). EN-UNE-133306 Terminología de mantenimiento. Madrid: AENOR.

Crespo, A., Sánchez, A., \& Moreu de León, P. (2004). Ingeniería de mantenimiento. Madrid: AENOR. 
Ebeling, C. (1997). Reliability and Maintainability Engineering. USA: McGraw Hill.

Escobar, L., Villa, E., \& Yáñez, S. (2003). Confiabilidad: Historia, estado del arte y desafíos futuros. Dyna, 70(140), 21.

Gasca, M., Camargo, L., \& Medina, B. (2017). Sistema para Evaluar la Confiabilidad de Equipos Críticos en el Sector Industrial. Información. Tecnológica, 26(4), 14.

Gómez, A. (2002). Manual del Ingeniero de Mantenimiento. México: Facilites.

Holmber, K., Folkeson, S., Berrgman, B., \& Ostvic, R. (1991). Operational reliability and systematic maintenance. New York: Elsevier Science Publishers LTD.

Knezevic, A. (1996). Mantenibilidad. Madrid: Isdefe.

Mors, L. (2009). Mantenimiento. Planeación, ejecución y control. México: Alfaomega.

Nachias, J. (1995). Fiabilidad. Madrid: Isdefe.

Parra, C., \& Crespo, A. (2014). Ingeniería de Mantenimiento y Fiabilidad Aplicada en la Gestión de Activos. Sevilla: Ingeman.

Parra, C., \& Crespo, A. (2016). Métodos de análisis de fiabilidad, mantenibilidad, disponibilidad y riego. Ingecom.

Ruiz, J. (2013). Propuesta metodológica para el cálculo y gestión de la confiabilidad del suministro energético en instalaciones. Medellín: Universidad Pontificia Bolivariana.

Yánez, M., Gómez, H., \& Valbuena, G. (2004). Ingeniería de la confiabilidad y análisis probabilístico del riesgo Reliability and risk management S.A.

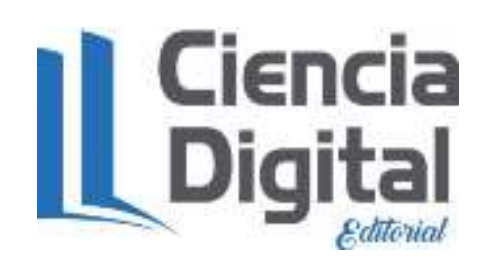


El artículo que se publica es de exclusiva responsabilidad de los autores y no necesariamente reflejan el pensamiento de la Revista Alfa Publicaciones.

\section{Ciencia
Digital
zethoul}

El artículo queda en propiedad de la revista y, por tanto, su publicación parcial y/o total en otro medio tiene que ser autorizado por el director de la Revista Alfa Publicaciones.
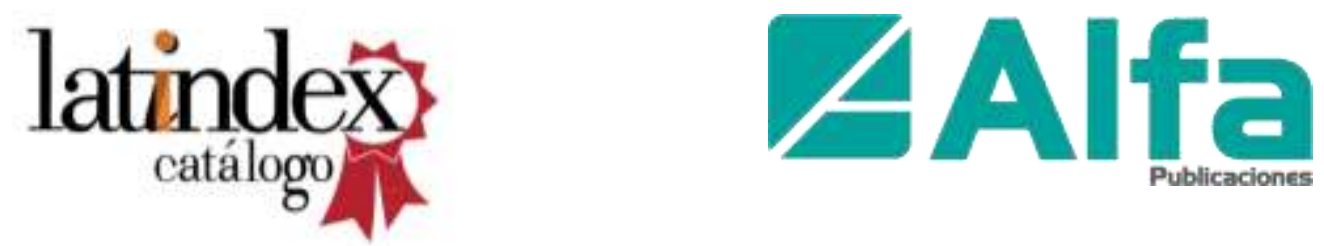

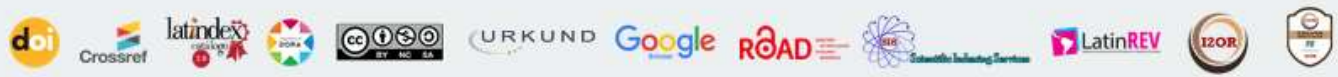

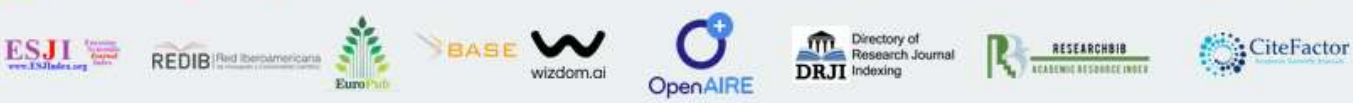

